\title{
Modeling and Optimal Control of a Class of Warfare Hybrid Dynamic Systems Based on Lanchester $(n, 1)$ Attrition Model
}

\author{
Xiangyong Chen ${ }^{1}$ and Ancai Zhang ${ }^{2}$ \\ ${ }^{1}$ School of Sciences, Linyi University, Linyi, Shandong 276005, China \\ ${ }^{2}$ School of Automobile Engineering, Linyi University, Linyi, Shandong 276005, China \\ Correspondence should be addressed to Xiangyong Chen; cxy8305@163.com
}

Received 13 December 2013; Revised 2 March 2014; Accepted 4 March 2014; Published 3 April 2014

Academic Editor: Rongni Yang

Copyright (c) $2014 \mathrm{X}$. Chen and A. Zhang. This is an open access article distributed under the Creative Commons Attribution License, which permits unrestricted use, distribution, and reproduction in any medium, provided the original work is properly cited.

For the particularity of warfare hybrid dynamic process, a class of warfare hybrid dynamic systems is established based on Lanchester equation in a $(n, 1)$ battle, where a heterogeneous force of $n$ different troop types faces a homogeneous force. This model can be characterized by the interaction of continuous-time models (governed by Lanchester equation), and discrete event systems (described by variable tactics). Furthermore, an expository discussion is presented on an optimal variable tactics control problem for warfare hybrid dynamic system. The optimal control strategies are designed based on dynamic programming and differential game theory. As an example of the consequences of this optimal control problem, we take the $(2,1)$ case and solve the optimal strategies in a $(2,1)$ case. Simulation results show the feasibility of warfare hybrid system model and the effectiveness of the optimal control strategies designed.

\section{Introduction}

In 1914, Lanchester [1] first proposed a mathematical model to describe and forecast quantitatively the development trend of battle. Since then, Lanchester equation has been used to analyze real wars $[2-5]$ and determine tactics for deploying forces in war game simulations, as they produce reasonably good predictions. The popularity and wide acceptance of the Lanchester models are due to their amenability to simple analysis and the fact that they, by and large, reflect the actual conflict situation. Undoubtedly, even in the modern hightech war, Lanchester equation can still make a comprehensive assessment and decision-making for a variety of battlefield factors.

To establish the mathematical model describing warfare process is the basis for researching quantitatively decisionmaking problems in conflicts. So far, warfare system models based on Lanchester equation have penetrated into many fields of military problems; the research core mainly focuses on extending and modifying Lanchester equation. For example, in [6], Sha introduced morale parameter based on the conventional Lanchester equation of casualty rate to set up mathematic models. In $[7,8]$, by introducing battlefield sensing coefficient and information superiority coefficient to modify the casualty rates of Lanchester equation, the generalized model for information warfare was proposed. In [9], considering the relationship between electronic jamming and operational efficiency, an expanded Lanchester square law model with variable efficiency factors was established. In $[10,11]$, a spatial modeling of Lanchester equations was conceptualized on the basis of explicit movement dynamics and balance of forces, ensuring stability and theoretical consistency with the original model. In $[12,13]$, some extensions of the Lanchester square law to inhomogeneous forces with an application to force allocation methodology were studied. However, all the models above have some limitations in war game simulations and tactical decision-making application, especially without regard to the interaction between the discrete event-driven tactics and the continuous force changes and without revealing better the complex operation mechanism, which is more close to the actual warfare dynamic process. 
In fact, warfare dynamic process is a hybrid dynamic process, which is characterized by the interaction of continuous time dynamic process (described, e.g., fighting strength changes) and discrete event dynamic process (described, e.g., fighting strength scheduling, variable tactics). Thus, how to establish warfare hybrid dynamic system model, which integrates the discrete events with continuous time, has become a problem to be solved urgently. In $[14,15], \mathrm{Xu}$ first proposed the modeling idea of warfare hybrid dynamic system; the basic frame and method to solve this kind of problems are investigated and a series of key technologies are emerged. Thus, in this paper, we first study the modeling of warfare hybrid dynamic system based on Lanchester equation. Introducing the discrete event variable, which can trigger the occurrence of variable tactics, the terms concerned with operational losses within Lanchester equation are modified, and a new warfare hybrid dynamic system is established.

Optimal control problem of warfare dynamic system has been an area of considerable research interest and has been an absolutely necessary tache on using Lanchester equation to research the tactic decision-making problem. So far, a wide variety of research achievements on this problem have been obtained, such as. In [16], Taylor considered a class of optimal resource allocation problems as a time sequential resource allocation problem and presented a solution in the optimal control framework based on Lanchester equation. In $[17,18]$, Taylor and Issacc used differential game theory to study the tactic decision-making problem, respectively. In $[19,20]$, Sha and Zeng and Li et al. solved the firepower assignment problem based on Lanchester equation and differential game theory, and they also validated, from another aspect, the principle of concentrating superior firepower in attack. In [21-25], optimal strategies of force resource complementary were obtained based on optimal control and differential game. However, the recent study results are confined to determine the optimal control problem of warfare continuous dynamic process. And there are not the effective theories and methods to solve the optimal tactic control problem of discrete event dynamic process. Thus, in this paper, we study the problem of building the optimal variable tactics control of warfare hybrid dynamic system based on Lanchester $(n, 1)$ model $[26,27]$. By using dynamic programming and differential game theory, we present the approach to obtain the optimal control strategies.

The paper is organized as follows. In Section 2, warfare hybrid dynamic process description and modeling are studied. In Section 3, the optimal control strategies of warfare hybrid dynamic system based on Lanchester $(n, 1)$ model are given, and the simulation results demonstrate the effectiveness of proposed optimal control schemes. And finally some concluding remarks are given in Section 4.

\section{Warfare Hybrid Dynamic Process Description and Modeling}

In order to directly understand the basic frame of warfare hybrid dynamic system, the evolution analysis of warfare process is given in Figure 1.
Firstly, let $X$ denote the attacking force and let $Y$ denote the defending force. It is assumed that the attacking force consists of one type of forces and the defending force consists of $n$ type of forces. Then we can make out that, from Figure 1, the composition of warfare hybrid dynamic process can be considered as the following key elements of two; they are the event-driven tactics and the continuous force strength changes. The variable tactics evolution process can be described as follows:

(1) firstly, $Y$ will concentrate all superior firepower to attack $X$ throughout the period of battle. However, at the time $t_{0}, X$ decides the initial encounter according to each original situation;

(2) at the time $t_{1}$, after surveys, $X$ detects the opponent's targets and motives and responds with variable tactics, and a new belligerent encounter accordingly is established;

(3) at the time $t_{2}$, after fresh surveys, $X$ responds with variable tactics for good and consequently establishes a new belligerent encounter;

(4) the above-mentioned process continues.

There is no difficulty in deducing the conclusion that variable tactics of the decision-maker $X$ happen at the discrete moment, which can lead to the changes of the belligerent encounter and structural changes of the system, shown in features of discrete event dynamic system.

The warfare process evolution also involves the continuous control of force strengths on both combat units. Based on decision-maker's instructions and detected situation, each combat unit adjusts the control variables with the purpose of changing the force strengths; however, the warfare task is certain herein. It falls into a category of continuous control process with the systematic structure unchanged. Therefore, warfare dynamic process can be considered as warfare hybrid dynamic system, which is that force strengths change on both sides from one continuous system to another via certain variable tactics (those that change combat encounter), and every variable tactic happens; the whole system operates following the later's rules.

Inspired by [21], we can give some reasonable assumptions as follows.

Assumption 1. $X$ and $Y$ have, respectively, one and $n$ combat units, $x_{1}(t)$ and $y_{j}(t)(j=1, \ldots, n)$ are the strengths of one and $j$ th combat unit on both sides surviving at time $t$, and the original states are $x_{1}(0)=x_{10}$ and $y_{j}(0)=y_{j 0}$.

Assumption 2. Suppose that variable tactics happen at time $t_{k}(1 \leq k \leq \infty)$, where $t_{k} \in\left[t_{0}, t_{f}\right], t_{0} \leq \cdots \leq \cdots \leq t_{f}$, and $t_{0}$ is the initial time. $e_{s}(s=1, \ldots, k, \ldots)$ is the discrete event variable happening at time $t_{s}$; then the route of variable tactics can be described as

$$
r=\left(\left(t_{1}, e_{1}\right), \ldots,\left(t_{s}, e_{s}\right)\right)
$$

where $\left(t_{s}, e_{s}\right)$ means the occurrence of variable tactics at time $t_{s}$. 


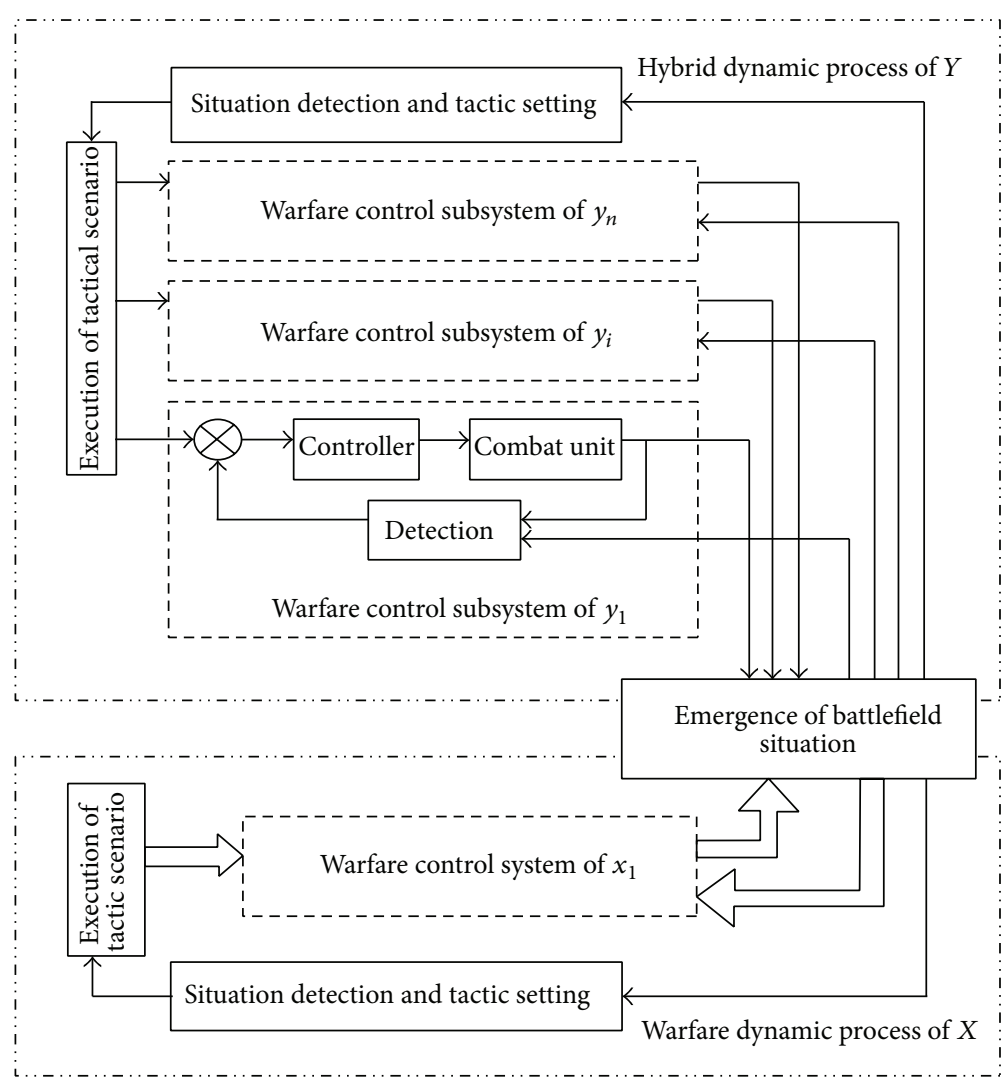

FIGURE 1: Evolution analysis of warfare hybrid dynamic process in a $(n, 1)$ battle.

Motivated by the above discussions, a class of warfare hybrid dynamic systems based on Lanchester equation can be established as follows:

$$
\begin{gathered}
\dot{x}_{1}(t)=-\sum_{j=1}^{n} \alpha_{j 1} \psi_{j 1_{e_{s}}} y_{j}(t)+u_{1}(t), \\
\dot{y}_{j}(t)=-\beta_{1 j} \phi_{1 j_{e_{s}}} x_{1}(t)+v_{j}(t),
\end{gathered}
$$

where $\beta_{1 j}$ is the nonnegative attrition coefficient of $x_{1}$ to $y_{j}$ and $\alpha_{j 1}$ is the nonnegative attrition coefficient of $y_{j}$ to $x_{1}$; $u_{1}(t)$ and $v_{j}(t)$ are corresponding control input; $\psi_{j 1_{e_{s}}} \in\{0,1\}$ and $\phi_{1 j_{e_{s}}} \in\{0,1\}$ are corresponding encounter tactics; then $\Psi_{e_{s}}$ and $\Phi_{e_{s}}$ are variable tactic matrices driven by discrete events:

$$
\Psi_{e_{s}}=\left[\begin{array}{llll}
\psi_{11_{e_{s}}} & \psi_{21_{e_{s}}} & \cdots & \psi_{n 1_{e_{s}}}
\end{array}\right] ; \quad \Phi_{e_{s}}=\left[\begin{array}{c}
\phi_{11_{e_{s}}} \\
\phi_{12_{e_{s}}} \\
\vdots \\
\phi_{1 n_{e_{s}}}
\end{array}\right] .
$$

From Assumption 2, it is known that the variable tactics driven by discrete events will satisfy

$$
\begin{gathered}
\Psi_{e_{s-1}} \times e_{s} \longrightarrow \Psi_{e_{s}} \\
\Phi_{e_{s-1}} \times e_{s} \longrightarrow \Phi_{e_{s}} .
\end{gathered}
$$

Remark 3. The values of switch variable $\psi_{j 1_{e_{s}}} \in\{0,1\}$ and $\phi_{1 j_{e_{s}}} \in\{0,1\}$ are given according to the encounter relation between two combat units on both sides:

$$
\begin{aligned}
& \psi_{j 1_{e_{s}}}= \begin{cases}0, & \text { No encounter between } y_{j} \text { and } x_{1}, \\
1, & y_{j} \text { encounters } x_{1} \text { with all forces, }\end{cases} \\
& \phi_{i j_{e_{s}}}= \begin{cases}0, & \text { No encounter between } y_{j} \text { and } x_{1}, \\
1, & x_{1} \text { encounters } y_{j} \text { with all forces. }\end{cases}
\end{aligned}
$$

Remark 4. $\Psi_{e_{s}}$ and $\Phi_{e_{s}}$ are driven to change by discrete event $e_{s}$, which therefore affects and changes system (2). If (4) is tenable, then continuous subsystems of $\Psi_{e_{s-1}}$ and $\Phi_{e_{s-1}}$ are changed to that of $\Psi_{e_{s}}$ and $\Phi_{e_{s}}$, which tell that variable tactics decide the number of continuous subsystems.

From Remarks 3 and 4, this model has a better description of the interaction of continuous-time models (governed by Lanchester equations) and of logic rules and discrete event systems (described, e.g., by variable tactics). And it is known that the discrete part makes the decision for the whole system to switch to another set of control rules if conditions are favorable, and the continuous part as a result works according to the new rules. 


\section{Optimal Control of Warfare Hybrid System via Lanchester $(n, 1)$ Model}

3.1. Problem Statement. In this section, the optimal variable tactics control problem of warfare hybrid dynamic system in a $(n, 1)$ battle, in which a heterogeneous force of $n$ different troop types faces a homogeneous force, is investigated. With what is mentioned above, we present some assumptions which will be used.

Assumption 5. The values of switch variables $\psi_{j 1_{e_{s}}}$ and $\phi_{1 j_{e_{s}}}$ satisfy the following conditions:

$$
\psi_{j 1_{e_{s}}}=1 ; \quad \sum_{j=1}^{n} \phi_{i j_{e_{s}}}=1
$$

Meanwhile, we suppose that the most effective battle stage is $[0, T]$, where $T$ is the end time of battle, $x_{1}(T)$ and $y_{j}(T)$ are the residual of strengths on both sides in the terminal time $T$, $x_{1}(T) \neq 0$, and $y_{j}(T) \neq 0$.

From Assumption 5, the system model is rewritten to be

$$
\begin{aligned}
& \dot{x}_{1}(t)=-\sum_{j=1}^{n} \alpha_{j 1} y_{j}(t)+u_{1}(t), \\
& \dot{y}_{j}(t)=-\beta_{1 j} \phi_{1 j_{e_{s}}} x_{1}(t)+v_{j}(t) .
\end{aligned}
$$

The objective function associated with system (6) is of the following form:

$$
J=\eta_{1} x_{1}(T)-\sum_{j=1}^{n} \theta_{j} y_{j}(T),
$$

where $\eta_{1}$ and $\theta_{j}$ are the relative operation indices, which is the weight of the importance of the corresponding units on both sides. Then, $\eta_{1} x_{1}(T)$ and $\sum_{j=1}^{n} \theta_{j} y_{j}(T)$ are the residual of actual strengths on both sides in the terminal time $T$.

Now, the optimal variable tactics control problem can be described as follows. The attacking side $X$ selects the number of tactics changes, the time of every variable tactics, and the sequences of corresponding variable tactics $\Phi_{e_{s}}^{*}(s=1, \ldots, k)$ to maximize the objective function $J$.

3.2. Solving Method for the Optimal Control Strategies. In this subsection, we analyze the conditions of the optimal variable tactics and give a quantitative analysis of the variable tactics process. Finally, a solving method for the optimal control strategies is designed.

For the above optimal control problem, we introduce the adjoint function as follows:

$$
\left[\begin{array}{l}
\lambda \\
\mu
\end{array}\right]=\left(\lambda_{1}, \mu_{1}, \ldots, \mu_{n}\right)^{T}
$$

and construct the Hamilton function to be

$$
\begin{aligned}
& H(x, y, \lambda, \mu, \Phi, t) \\
& =-\sum_{j=1}^{n}\left(\lambda_{1} \alpha_{j 1}\right) y_{j}-\sum_{j=1}^{n} \mu_{j} \beta_{1 j} \phi_{1 j e_{s}} x_{1} \\
& \quad+\lambda_{1} u_{1}(t)+\sum_{j=1}^{n} \mu_{j} v_{j}(t) .
\end{aligned}
$$

Then, by using the Minimax principle of differential game, the necessary conditions about the optimal tactics are that there exist the corresponding adjoint functions $\lambda^{*}(t)$ and $u^{*}(t)$, which satisfy

$$
\begin{gathered}
\dot{\lambda}_{1}(t)=-\frac{\partial H}{\partial x_{1}}=\sum_{j=1}^{n} \mu_{j} \beta_{i j} \phi_{i j_{e_{s}}}, \\
\lambda_{1}(T)=\frac{\partial J}{\partial x_{1}}=\eta_{1}, \\
\dot{\mu}_{j}(t)=-\frac{\partial H}{\partial y_{j}}=\lambda_{1} \alpha_{j 1}, \\
\mu_{j}(T)=\frac{\partial J}{\partial y_{j}}=-\theta_{j} .
\end{gathered}
$$

From (11), we have

$$
\begin{array}{ll}
\dot{\lambda}_{1}(t)<0, & \dot{\mu}_{j}(t)>0, \\
\lambda_{1}(T)>0, & \mu_{j}(T)<0 .
\end{array}
$$

Therefore, for any time $t \in[0, T]$, it is easy to get

$$
\lambda_{1}(t)>0, \quad \mu_{j}(t)<0 .
$$

Then, we can obtain that

$$
\begin{aligned}
& H\left(x^{*}, y^{*}, \lambda^{*}, \mu^{*}, \Phi_{e_{s}}^{*}, t\right) \\
& =\max _{\Phi_{e_{s}}} H\left(x^{*}, y^{*}, \lambda^{*}, \mu^{*}, \Phi_{e_{s}}, t\right) \\
& =\max _{\Phi_{e_{s}}}\left(\sum_{j=1}^{n}-\mu_{j}^{*} \beta_{1 j} \phi_{1 j_{e_{s}}} x_{1}^{*}\right) .
\end{aligned}
$$

From (14) and $x_{1}^{*}>0$, we know that $\sum_{j=1}^{n}\left(-\mu_{j}^{*}\right) \beta_{i j} \phi_{i j_{e_{s}}}$ is the weighted average of $-\mu_{j}^{*} \beta_{i j}$; then the optimal tactics satisfy

$$
\phi_{i j_{e_{s}}}^{*}=\left\{\begin{array}{l}
1, \quad \max _{j}\left(-\mu_{j}^{*} \beta_{1 j}\right)=-\mu_{j^{*}}^{*} \beta_{1 j^{*}}, \\
0, \quad \max _{j}\left(-\mu_{j}^{*} \beta_{1 j}\right) \neq-\mu_{j^{*}}^{*} \beta_{1 j^{*}} .
\end{array}\right.
$$

Remark 6. Since $\mu_{j}(T)$ is a continuous function, $\mu_{j}^{*}(T) \beta_{1 j}$ is also continuous; thus, the optimal tactic strategy $\phi_{i j_{e_{s}}}^{*}$ remains stable on a period of time; that is, $\phi_{i j_{e_{s}}}^{*}$ remains stable at the time interval $[T-\delta, T]$. 
Based on the above analysis, we discuss the variable tactics process about the attacking side $X$. And the existence conditions of variable tactics are investigated in the following theorem.

Theorem 7. If there exist at least two functions $\mu_{j_{l}}(t) \beta_{1 j_{l}}$ and $\mu_{j_{g}}(t) \beta_{1 j_{g}}\left(l, g \in\{1, \ldots, n\}\right.$ and $\left.j_{l} \neq j_{g}\right)$ at the time interval $\left[\Delta_{k}, T\right]$, such that

$$
\begin{gathered}
-\mu_{j_{l}}(t) \beta_{1 j_{l}}<-\mu_{j_{g}}(t) \beta_{1 j_{g}}, \\
-\mu_{j_{l}}\left(\Delta_{k}\right) \beta_{1 j_{l}}=-\mu_{j_{g}}\left(\Delta_{k}\right) \beta_{1 j_{g}}, \\
-\dot{\mu}_{j_{l}}\left(\Delta_{k}\right) \beta_{1 j_{l}}<-\dot{\mu}_{j_{g}}\left(\Delta_{k}\right) \beta_{1 j_{g}},
\end{gathered}
$$

hold, then one obtains that

$$
-\mu_{j_{l}}(t) \beta_{1 j_{l}}>-\mu_{j_{g}}(t) \beta_{1 j_{g}}
$$

at the left neighborhood of $t=\Delta_{k}$. That is, there exists a variable tactic for $X$ at $[t, T]\left(t<\Delta_{k}\right)$, and $\Delta_{k}$ is the minimum time of tactic change.

Proof. From (15), it is not difficult to show that

$$
\phi_{i j_{e_{s}}^{*}}^{*}=\left\{\begin{array}{l}
1, \quad \max _{j}\left(-\mu_{j}^{*}(T) \beta_{1 j}\right)=-\mu_{j^{*}}^{*}(T) \beta_{1 j^{*}}, \\
0, \quad \max _{j}\left(-\mu_{j}^{*}(T) \beta_{1 j}\right) \neq-\mu_{j^{*}}^{*}(T) \beta_{1 j^{*}} .
\end{array}\right.
$$

So, there exist at least two functions $\mu_{j_{l}}(t) \beta_{1 j_{l}}$ and $\mu_{j_{g}}(t) \beta_{1 j_{g}}\left(j_{l} \neq j_{g}\right)$ at the time interval $\left[\Delta_{k}, T\right]$, which satisfy

$$
-\mu_{j_{l}}(t) \beta_{1 j_{l}}<-\mu_{j_{g}}(t) \beta_{1 j_{g}} .
$$

From (18), we have that

$$
\begin{aligned}
& \lim _{\varepsilon^{-} \rightarrow 0} \frac{-\mu_{j_{l}}\left(\Delta_{k}\right) \beta_{1 j_{l}}-\left(-\mu_{j_{l}}\left(\Delta_{k}-\varepsilon\right) \beta_{1 j_{l}}\right)}{\varepsilon} \\
& \quad<\lim _{\varepsilon^{-} \rightarrow 0} \frac{-\mu_{j_{g}}\left(\Delta_{k}\right) \beta_{1 j_{g}}-\left(-\mu_{j_{g}}\left(\Delta_{k}-\varepsilon\right) \beta_{1 j_{g}}\right)}{\varepsilon} .
\end{aligned}
$$

Combining the aforementioned inequality with (17) yields

$$
-\mu_{j_{l}}\left(\Delta_{k}-\varepsilon\right) \beta_{1 j_{l}}>-\mu_{j_{g}}\left(\Delta_{k}-\varepsilon\right) \beta_{1 j_{g}} .
$$

Since the following inequality

$$
-\mu_{j_{l}}\left(\Delta_{k}+\varepsilon\right) \beta_{i j_{l}}<-\mu_{j_{g}}\left(\Delta_{k}+\varepsilon\right) \beta_{i j_{g}}
$$

holds at the right neighborhood of $t=\Delta_{k}$, therefore, there exists a variable tactic for $X$ at the time $\Delta_{k}\left(0<\Delta_{k}<T\right)$.

Now, we will investigate that $\Delta_{k}$ is the minimum time of tactic change. Firstly, we suppose that $\Delta_{k 1}\left(\Delta_{k 1}<\Delta_{k}\right)$ is the minimum time of tactic change; then we have

$$
\begin{aligned}
& -\mu_{j_{l}}\left(\Delta_{k 1}+\varepsilon\right) \beta_{1 j_{l}}<-\mu_{j_{g}}\left(\Delta_{k 1}+\varepsilon\right) \beta_{1 j_{g}}, \\
& -\mu_{j_{l}}\left(\Delta_{k 1}-\varepsilon\right) \beta_{1 j_{l}}>-\mu_{j_{g}}\left(\Delta_{k 1}-\varepsilon\right) \beta_{1 j_{g}} .
\end{aligned}
$$

From (17), (18), and (19), we easily get

$$
-\mu_{j_{l}}(t) \beta_{1 j_{l}}<-\mu_{j_{g}}(t) \beta_{1 j_{g}}, \quad t \in\left(\Delta_{k 1}, T\right] ;
$$

then there exists the contradiction between (23) and (26). Thus, $\Delta_{k}$ is the minimum time of tactic change when it draws near the termination time $T$. The proof is completed.

According to Theorem 7, we give a solving method of the optimal control strategies.

Step 1. Using $\mu_{j}(T) \beta_{1 j}$, we solve the optimal tactics $\Phi_{e_{k}}$ at the time interval near the termination time $T$. Furthermore, we seek the minimum time of tactic change $\Delta_{k}$, which satisfies Theorem 7.

Step 2. Using $\mu_{j}\left(\Delta_{k}\right) \beta_{1 j}$, the optimal tactics $\Phi_{e_{k-1}}$ are obtained at the time interval near $\Delta_{k}$, and we seek the minimum time of tactic change $\Delta_{k-1}$

Step 3. The aforementioned process continues. When the conditions of tactic change cannot hold in the time interval $\left[0, \Delta_{1}\right]$ near the initial time, the solving process stops.

Step 4. Sorting $\Delta_{1}, \ldots, \Delta_{k}$, we obtain that the discrete event variable $e=\left\{e_{1}, \ldots, e_{f}\right\}(0 \leq f<\infty)$ and the route of variable tactic $r=\left(\left(t_{1}, e_{1}\right), \ldots,\left(t_{f}, e_{f}\right)\right)$, where, $t_{f} \in$ $\left\{\Delta_{1}, \ldots, \Delta_{k}\right)$ is the time of tactic change and $e_{f}$ is a discrete event.

3.3. Application Example Analysis. As an example of the consequences of the optimal control problem, we take the $(2,1)$ case and solve the optimal strategies in a $(2,1)$ case. In this subsection, we consider the warfare dynamic system model that is described by

$$
\begin{gathered}
\dot{x}_{1}=-\sum_{j=1}^{2} \alpha_{j 1} y_{j}+u_{1}, \\
\dot{y}_{1}=-\beta_{11} \phi_{11_{e_{k}}} x_{1}+v_{1}, \\
\dot{y}_{2}=-\beta_{12} \phi_{12_{e_{k}}} x_{1}+v_{2},
\end{gathered}
$$

where $x_{1}(t), y_{1}(t)$, and $y_{2}(t)$ are the strengths of two opposing forces surviving at time $t$.

The objective function associated with the system (27) is of the following form:

$$
J=\eta_{1} x_{1}-\theta_{1} y_{1}-\theta_{2} y_{2}
$$

The relevant parameters of system are as follows. The initial force strengths are $x_{10}=100, y_{10}=30, y_{20}=30 ; \alpha_{11}=$ 9, $\alpha_{21}=1, \beta_{11}=\beta_{12}=1$ are the nonnegative attrition coefficients; the battle terminal time is $T=0.489$; the relative operation indices are $\eta_{1}=9, \theta_{1}=1, \theta_{2}=9$, and we choose that $u_{1}=0, v_{1}=v_{2}=0$. In the proposed solving algorithm, we set the initial values $t_{0}=T, \lambda_{1}(T)=9, \mu_{1}(T)=-1$, and $\mu_{2}(t)=-9$ and the step length $\kappa=0.001$; then we can know that $t=t_{0}-\chi \kappa$, where $\chi$ is the cycle number. 


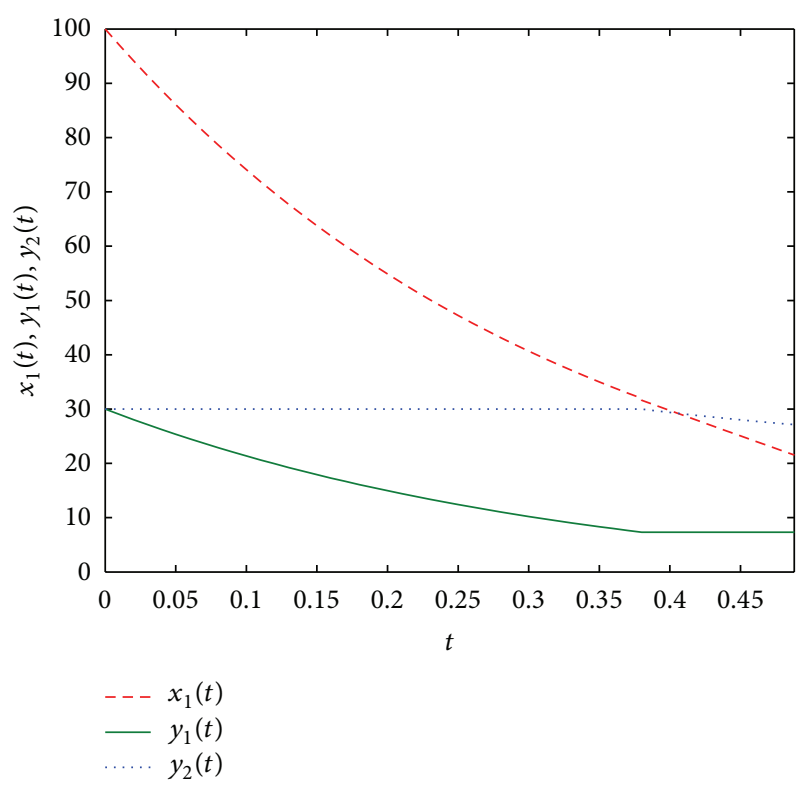

FIGURE 2: Strength change curves of each combat unit on both sides.

Solving the optimal control problem by Matlab Toolbox yields that when $\chi=105$, the variable tactics occur, and the corresponding time is $\Delta_{1}=0.384$; then it is easy to get that the optimal tactics of $X$ are

$$
\begin{array}{lll}
\phi_{11_{e_{k}}}(t)=1, & \phi_{12_{e_{k}}}(t)=0, & 0<t \leq \Delta_{1}, \\
\phi_{11_{e_{k}}}(t)=0, & \phi_{12_{e_{k}}}(t)=1, & \Delta_{1}<t \leq T .
\end{array}
$$

So the discrete event variable is $e_{1}=\left\{\Phi_{e_{0}}, \Phi_{e_{1}}\right\}$, where $\Phi_{e_{0}}=$ $\left[\begin{array}{l}1 \\ 0\end{array}\right] ; \Phi_{e_{1}}=\left[\begin{array}{l}0 \\ 1\end{array}\right]$. Therefore, the solution of optimal control problem in a $(2,1)$ battle can be gotten as follows. $X$ has a variable tactic in $\Delta_{1}=0.384$, and the optimal tactics $\Phi_{e_{s}}^{*}$ for $X$ satisfy

$$
\begin{array}{lll}
\phi_{11_{e_{0}}}^{*}(t)=1 ; & \phi_{12_{e_{0}}}^{*}(t)=0, & 0<t \leq \Delta_{1}, \\
\phi_{11_{e_{0}}}^{*}(t)=0 ; & \phi_{12_{e_{1}}}^{*}(t)=1, & \Delta_{1} \leq t \leq T .
\end{array}
$$

And the route of variable tactic $r=\left(\left(0, e_{0}\right), \ldots,\left(\Delta_{1}, e_{1}\right)\right)$, and the optimal value of $J$ is $J^{*}=44.152$.

Figure 2 shows the change of state trajectories of the units on both warring sides. It is easy to see that the state values change in $t=\Delta_{1}$; meanwhile, the state changes are nonnegative, and the change of variable tactics always holds in the time interval $[0, T]$.

\section{Conclusions}

In this paper, we established a class of warfare hybrid dynamic systems based on Lanchester equation in a battle between an attacker with one type of force and a defender with $n$ types of forces. For the attacking side, an optimal control problem of warfare hybrid dynamic system in a $(n, 1)$ battle was investigated. Then the optimum condition and the solving method about the game problem are given. Simulation results illustrate the effectiveness of proposed optimal strategies. This is of great significance in analyzing quantitatively military actions. However, the proposed warfare hybrid dynamic model in this paper fails to consider the warfare dynamic process in a $(n, m)$ battle, in which an attacker has $m$ type of forces and a defender has $n$ types of forces. Thus, constructing a more reasonable model and employing advanced control techniques to investigate the warfare dynamic game problem in a $(n, m)$ battle are our future research directions.

\section{Conflict of Interests}

The authors declare that there is no conflict of interests regarding the publication of this paper.

\section{Acknowledgments}

The authors would like to express their deepest gratitude to Dr. Qiu Jianlong, Dr. Jianwei Zhou, and Dr. Chen Xiao for their helpful suggestions on the English writing of the revised version. This work was supported in part by the Applied Mathematics Enhancement Program (AMEP) of Linyi University and the National Natural Science Foundation of China, under Grant nos. 61273012, 11301252, 61304023, and 11201212, and by a Project of Shandong Province Higher Educational Science and Technology Program under Grant nos. J13LI11 and J12LI58.

\section{References}

[1] F. W. Lanchester, Aircraft in Warfare: The Dawn of the Fourth Arm, Constable, London, UK, 1916, Lanchester Press, 1999.

[2] T. W. Lucas and T. Turkes, "Fitting Lanchester equations to the battles of Kursk and Ardennes," Naval Research Logistics, vol. 51, no. 1, pp. 95-116, 2004.

[3] J. H. Engel, "A verification of Lanchester's law," Journal of the Operations Research Society of America, vol. 2, no. 2, pp. 163171, 1954.

[4] T. W. Lucas and J. A. Dinges, "The effect of battle circumstances on fitting Lanchester equations to the battle of Kursk," Military Operations Research, vol. 9, no. 2, pp. 17-30, 2004.

[5] I. R. Johnson and N. J. MacKay, "Lanchester models and the battle of Britain," Naval Research Logistics, vol. 58, no. 3, pp. 210222, 2011.

[6] J. C. Sha, Mathematic Tactics, Science Press, Beijing, China, 2003.

[7] J. Wang, F. Yang, Y. Liang et al., "Generalized Lanchester combat model for information war," Fire Control and Command Control, vol. 35, no. 2, pp. 50-53, 2010.

[8] X. Chen, Y. Jing, C. Li, and M. Li, "Warfare command stratagem analysis for winning based on Lanchester attrition models," Journal of Systems Science and Systems Engineering, vol. 21, no. 1, pp. 94-105, 2012.

[9] Y. Wang, T. Sun, X.-W. Li, and Q. Pan, "Modeling and simulation of combating process based on Lanchester equation," Journal of Systems Engineering and Electronics, vol. 31, no. 7, pp. 1677-1679, 2009. 
[10] T. Keane, "Combat modelling with partial differential equations," Applied Mathematical Modelling, vol. 35, no. 6, pp. 27232735, 2011.

[11] E. González and M. Villena, "Spatial Lanchester models," European Journal of Operational Research, vol. 210, no. 3, pp. 706-715, 2011.

[12] D. M. Roberts and D. M. Conolly, "An extension of the Lanchester square law to inhomogeneous forces with an application to force allocation methodology," Journal of the Operational Research Society, vol. 43, no. 8, pp. 741-752, 1992.

[13] R. K. Colegrave and J. M. Hyde, "The Lanchester square-law model extended to a $(2,2)$ conflict," IMA Journal of Applied Mathematics, vol. 51, no. 2, pp. 95-109, 1993.

[14] X. H. Xu, H. Wang, and C. M. Xu, "Computer games are an efficient tool for event game theory," in Proceedings of the 22nd Chinese Control and Decision Conference (CCDC '11), pp. 343633441, Mianyang, China, May 2011.

[15] X. H. Xu and H. Y. Shi, "A case study: air fight problem and hybrid game," in Proceedings of 19th Chinese Control and Decision Conference (CCDC '11), pp. 913-916, Northeastern University Press, Shenyang, China, 2007.

[16] J. G. Taylor, "Lanchester-type models of warfare and optimal control," Naval Research Logistics Quarterly, vol. 21, pp. 79-106, 1974.

[17] R. P. Issacc, Differential Game, John Wiley, New York, NY, USA, 1965.

[18] J. G. Taylor, "Differential-game examination of optimal timesequential fire-support strategies," Naval Research Logistics Quarterly, vol. 25, no. 2, pp. 323-355, 1978.

[19] J. C. Sha and A. J. Zeng, "Research on the warfare theory of Lanchester and tactics," in Proceeding of Control and Decision Conference of China, pp. 1134-1136, Xiamen, China, 1994.

[20] D.-F. Li, T. Sun, and Y.-C. Wang, "Differential game model and its solution for the firepower-assignment in vessel formations in information war," Control Theory and Applications, vol. 25, no. 6, pp. 1163-1166, 2008.

[21] R. L. Helmbold, "Direct and inverse solution of the Lanchester square law with general reinforcement schedules," European Journal of Operational Research, vol. 77, no. 3, pp. 486-495, 1994.

[22] H.-M. Chen, "An optimal control problem in determining the optimal reinforcement schedules for the Lanchester equations," Computers \& Operations Research, vol. 30, no. 7, pp. 1051-1066, 2003.

[23] X. Y. Chen, Y. W. Jing, C. J. Li, and X. P. Liu, “Optimal strategies for winning in military conflicts based on the Lanchester equation," Control and Decision, vol. 26, no. 6, pp. 945-948, 2011.

[24] D. F. Li, A. S. Tan, and F. Luo, "Optimization model of reinforcements based on differential game and its solving method," Operations Research and Management Science, vol. 11, no. 4, pp. 16-20, 2002.

[25] X. Y. Chen, Y. W. Jing, C. J. Li et al., "Differential game model and its solutions for force resource complementary via Lanchester square law equation," in Proceeding of the 18th IFAC World Congress, pp. 1024-1030, Milano, Italy, 2011.

[26] G. T. Kaup, D. J. Kaup, and N. M. Finkelstein, “The Lanchester $(n, 1)$ problem," Journal of the Operational Research Society, vol. 56, no. 12, pp. 1399-1407, 2005.

[27] P. S. Sheeba and D. Ghose, "Optimal resource partitioning in conflicts based on lanchester $(n, 1)$ attrition model," in Proceedings of the American Control Conference, pp. 638-643, Minneapolis, Minn, USA, June 2006. 


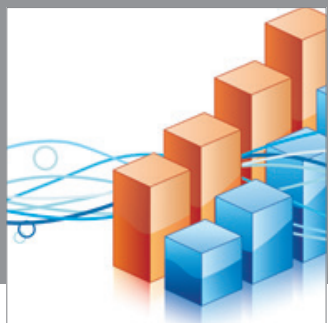

Advances in

Operations Research

mansans

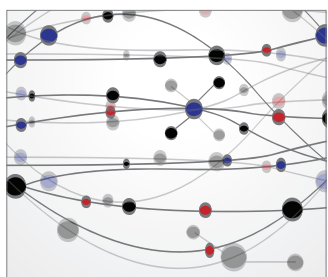

The Scientific World Journal
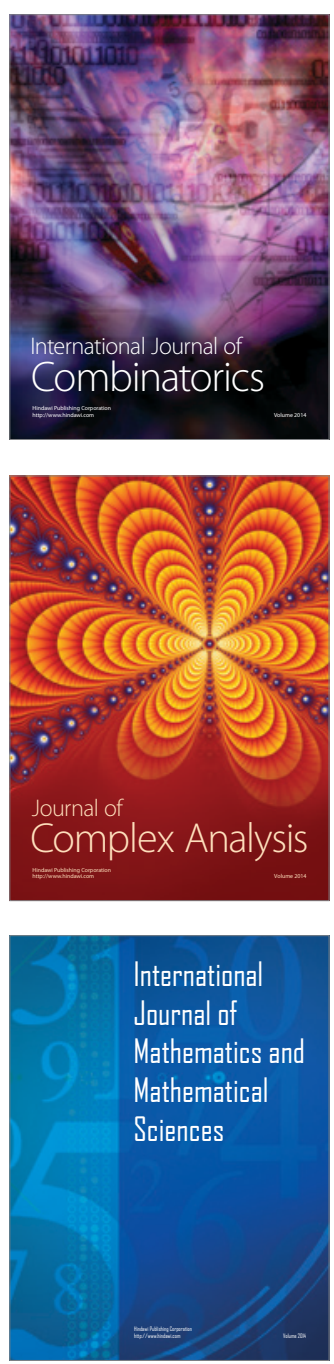
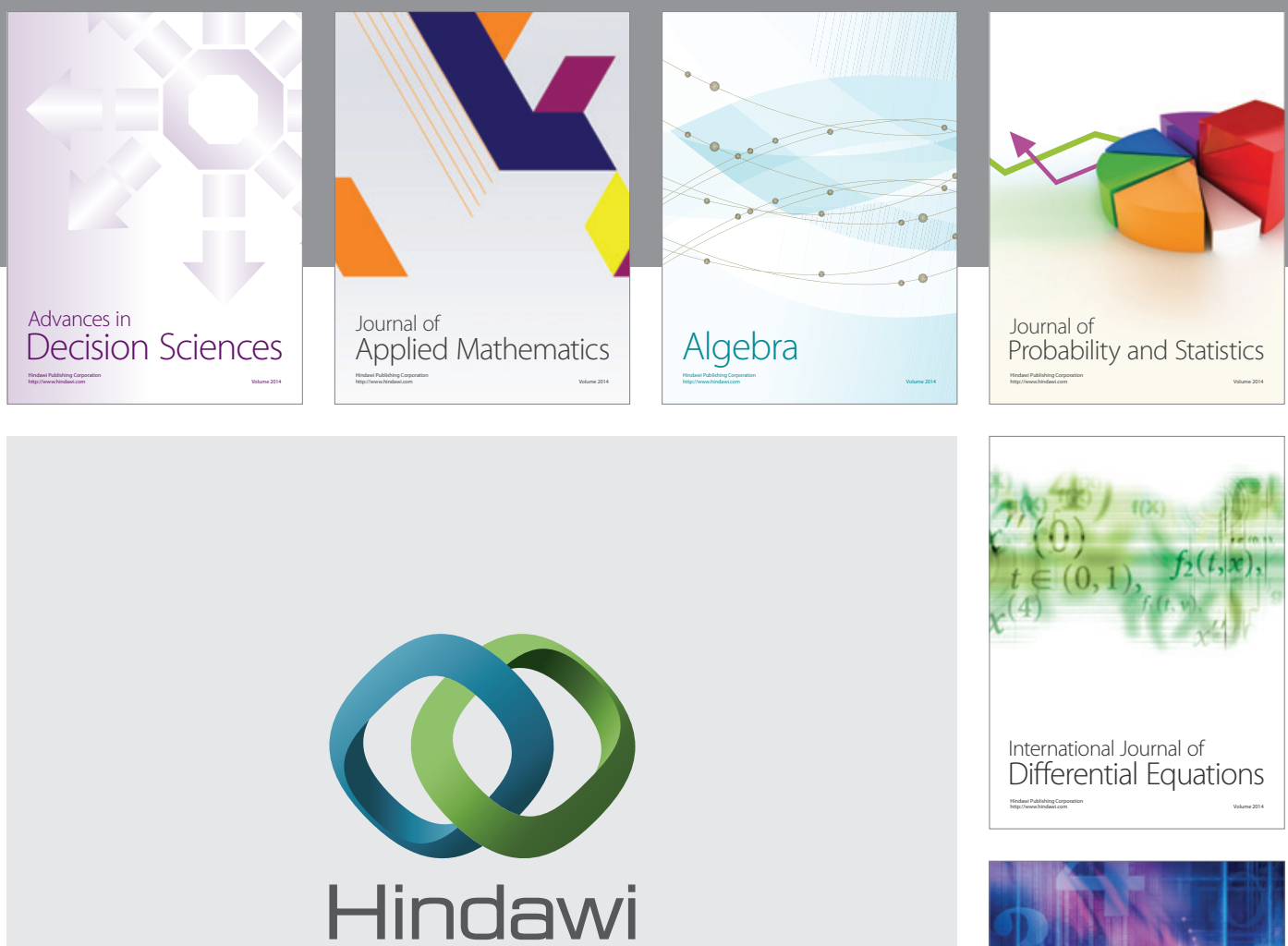

Submit your manuscripts at http://www.hindawi.com
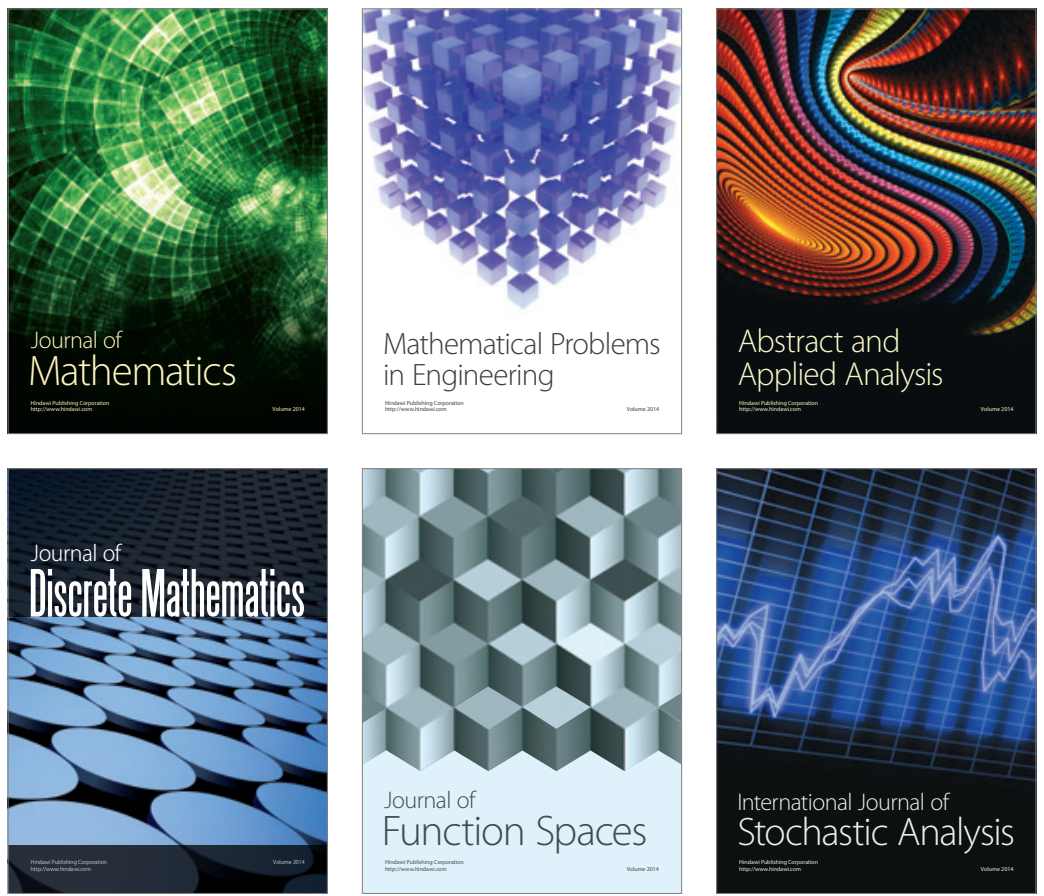

Journal of

Function Spaces

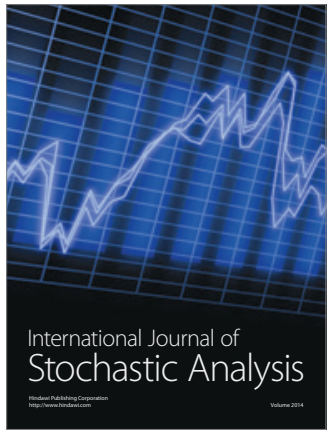

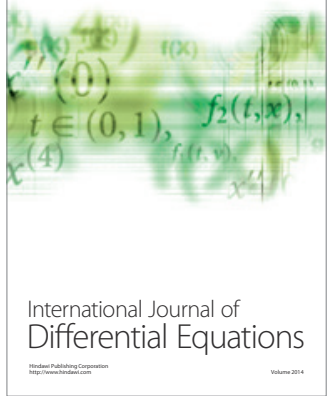
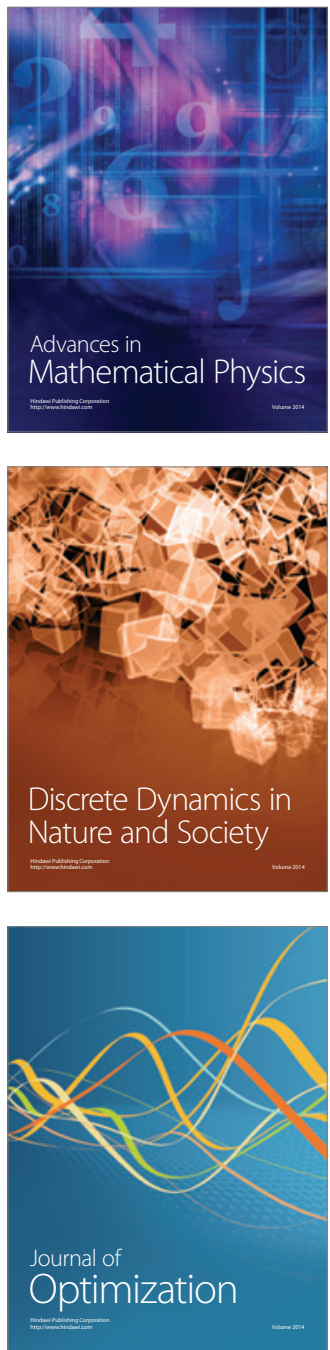